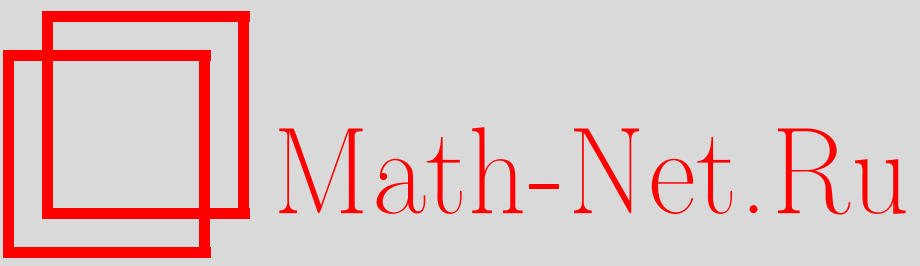

В. В. Пикуль, А. А. Ратников, Математическое моделирование процесса формирования трёхлойной цилиндрической оболочки из стеклометаллокомпозита, Вестн. Сам. гос. техн. ун-та. Сер. Физ.-мат. науки, 2010, выпуск 5(), 143-151

DOI: https://doi.org/10.14498/vsgtu808

Использование Общероссийского математического портала Math-Net.Ru подразумевает, что вы прочитали и согласны с пользовательским соглашением http://www . mathnet.ru/rus/agreement

Параметры загрузки:

IP : 3.81 .55 .215

26 апреля 2023 г., 18:18:10 
УДК 539.3

\title{
МАТЕМАТИЧЕСКОЕ МОДЕЛИРОВАНИЕ ПРОЦЕССА ФОРМИРОВАНИЯ ТРЁХСЛОЙНОЙ ЦИЛИНДРИЧЕСКОЙ ОБОЛОЧКИ ИЗ СТЕКЛОМЕТАЛЛОКОМПОЗИТА
}

\author{
В. В. Пикуль ${ }^{1}$, А. А. Ратников ${ }^{2}$ \\ 1 Институт проблем морских технологий ДВО РАН, \\ 690091, Владивосток, ул. Суханова, 5 а. \\ 2 Дальневосточный государственный технический университет \\ (ДВПИ им. В.В. Куйбышева), Естественно-научный институт, \\ Механико-математический факультет, \\ 690600, Владивосток, ул. Пушкинская, 10. \\ E-mails: pikulv@mail.ru, ratnikov2003@pisem.net
}

\begin{abstract}
Представлены результаты математического моделирования процесса изготовления иилиндрической оболочки из слоистого материала на основе силикатного стекла. Рассмотрены задачи остывания композитной оболочки и осесимметричного деформирования в прочессе её бормирования. Полученные результаты свидетельствуют о возможности изготовления трёхслойньх ицлиндрических оболочек из стеклометаллокомпозита, которые могут использоватъся для создания прочных корпусов глубоководной техники. Получены пределъные значения остаточных напряжений на поверхностях сопряжения металлических обшивок со стеклянным слоем, которые существенно менъше пределов прочности соединяемых материалов композитной оболочки.
\end{abstract}

Ключевые слова: иилиндрическая оболочка, стеклометаллокомпозит, наноматериал, температурная задача, теплопроводность, численный метод, дебормаицонная задача, аналитический метод, остаточные напряжения.

Введение. Стеклометаллокомпозит представляет собой композиционный материал, состоящий из стеклянного слоя, заключённого между металлическими обшивками [1]. В процессе его изготовления обеспечивается надёжное соединение стеклянного слоя с обшивками и создаются необходимые условия для формирования стеклянного слоя с наноструктурой без поверхностных микротрещин [2]. В результате стеклянный слой и стеклометаллокомпозит в целом приобретают исключительно высокую ударостойкость и прочность при относительно малой массе. И что очень важно - нет реальных препятствий для изготовления крупногабаритных изделий из стеклометаллокомпозита. Исследования эффективности стеклометаллокомпозита в глубоководной технике показали, что из него могут быть изготовлены прочные корпуса, способные обеспечить необходимую плавучесть глубоководной технике вплоть до предельных глубин Мирового океана [2]. При этом стоимость прочного корпуса из стеклометаллокомпозита оказывается намного ниже стоимости титанового корпуса.

В процессе формирования цилиндрической оболочки из стеклометаллокомпозита расплав стекломассы, имеющий начальную температуру свыше $1000^{\circ} \mathrm{C}$, остывает до нормальной температуры $\left(20^{\circ} \mathrm{C}\right)$. При понижении температуры стекломасса из вязкого состояния переходит в вязко-пластическое,

Владимир Васильевич Пикуль (д.ф.-м.н., проф.), зав. лабораторией, лаб. проблем прочности глубоководной техники. Александр Александрович Ратников, старший преподаватель, каф. прикладной математики и механики. 
и затем, наконец, в твёрдое состояние. Формирование цилиндрической композитной оболочки происходит под воздействием осесимметричного поля изменения температуры во времени. Изменения температуры, вызываемые деформированием стеклометаллокомпозита в процессе остывания, пренебрежимо малы по сравнению с температурой стекломассы. Вследствие этого общая задача формирования цилиндрической оболочки распадается на две части: температурную и деформационную. Поскольку потери тепла при остывании оболочки намного превосходят образующееся при её деформировании количество теплоты, температурная часть задачи решается независимо от деформационной. Решение деформационной задачи описано в работе [3], в данной статье приводится решение тепловой задачи и производится обобщение всего процесса формирования цилиндрической оболочки из стеклометаллокомпозита.

Процесс остывания стеклометаллокомпозита разбивается на 4 этапа:

- от температуры заливки стекла до температуры его соединения с металлическими облицовками;

- от температуры соединения с облицовками до температуры стеклования;

- отжиг при температуре стеклования;

- остывание от температуры стеклования до температуры окружающей среды.

Основной целью температурной части задачи является определение температурного режима формирования цилиндрической оболочки из стеклометаллокомпозита.

1. Математическая модель остывания цилиндрической оболочки. Остывание оболочки рассматривается в полярной системе координат. Основные координатные поверхности являются круговыми цилиндрическими. За их координаты приняты отсчитываемое от срединного поперечного сечения расстояние $s$ вдоль образующей, угол $\phi$ между начальным и текущим радиальными направлениями и координата $r$, отсчитываемая от оси вращения оболочки. Нумерация слоёв ведётся от внутреннего слоя. На рис. 1 показана часть оболочки в продольном разрезе.

Математическая модель процесса остывания цилиндрической оболочки из стеклометаллокомпозита состоит из уравнений теплопроводности, условий сопряжения слоёв, краевых и начальных условий.

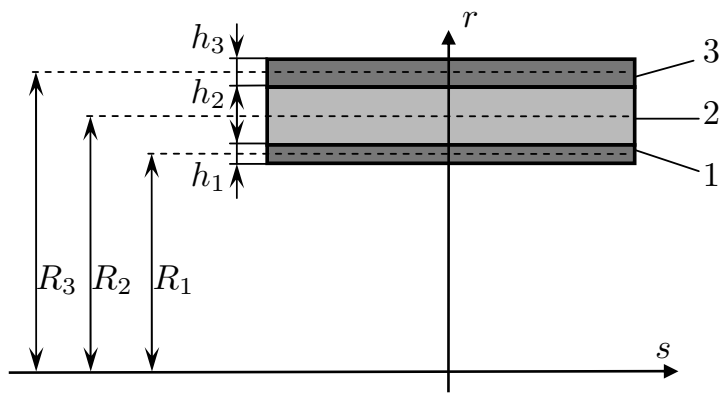

Рис. 1. Часть оболочки в продольном разрезе: 1-внутренняя металлическая обшивка; 2 - стеклянный слой; 3 - внешняя металлическая обшивка 
Процесс остывания является симметричным относительно среднего поперечного сечения оболочки и осесимметричным относительно оси симметрии, поэтому рассматривается только половина длины оболочки, а распределение поля температур оказывается независимым от координаты $\phi$.

Нелинейные уравнения теплопроводности для всех слоёв стеклометаллокомпозита записываются в виде

$$
\frac{\partial T_{k}}{\partial t}=\frac{a_{k}\left(T_{k}\right)}{\lambda_{k}\left(T_{k}\right)}\left[\frac{\partial}{\partial s}\left(\lambda_{k}\left(T_{k}\right) \frac{\partial T_{k}}{\partial s}\right)+\frac{1}{r} \frac{\partial}{\partial r}\left(\lambda_{k}\left(T_{k}\right) r \frac{\partial T_{k}}{\partial r}\right)\right]
$$

где $a_{k}\left(T_{k}\right)$ и $\lambda_{k}\left(T_{k}\right)$ - коэффициенты температуропроводности и теплопроводности $k$-того слоя оболочки, которые зависят от температуры $T_{k}$ в нём; $t-$ время.

Связь температурных полей между стеклозаполнителем и обшивками осуществляется посредством условий сопряжения по поверхностям контакта:

$$
\begin{gathered}
\left.T_{1}\right|_{r=R_{1}+h_{1} / 2}=\left.T_{2}\right|_{r=R_{2}-h_{2} / 2} ; \\
\left.\lambda_{1}\left(T_{1}\right) \frac{\partial T_{1}}{\partial r}\right|_{r=R_{1}+h_{1} / 2}+\delta q^{-}=\left.\lambda_{2}\left(T_{2}\right) \frac{\partial T_{2}}{\partial r}\right|_{r=R_{2}-h_{2} / 2} \\
\left.T_{2}\right|_{r=R_{2}+h_{2} / 2}=\left.T_{3}\right|_{r=R_{3}-h_{3} / 2} ; \\
\left.\lambda_{2}\left(T_{2}\right) \frac{\partial T_{2}}{\partial r}\right|_{r=R_{2}+h_{2} / 2}=\left.\lambda_{3}\left(T_{3}\right) \frac{\partial T_{3}}{\partial r}\right|_{r=R_{3}-h_{3} / 2}+\delta q^{+},
\end{gathered}
$$

где $\delta q^{-}$и $\delta q^{+}$- количество тепловой энергии, требующееся для обеспечения соединения стеклянного слоя с металлическими обшивками, происходящего за счёт их взаимной диффузии.

Граничные условия характеризуют закон теплообмена между поверхностью тела и окружающей средой при постоянном потоке тепла:

$$
\begin{aligned}
& \left.\lambda_{1}\left(T_{1}\right) \frac{\partial T_{1}}{\partial r}\right|_{r=R_{1}-h_{1} / 2}=\vartheta\left(\left.T_{1}\right|_{r=R_{1}-h_{1} / 2}-T_{c}\right) ; \\
& \left.\lambda_{3}\left(T_{3}\right) \frac{\partial T_{3}}{\partial r}\right|_{r=R_{3}+h_{3} / 2}=-\vartheta\left(\left.T_{3}\right|_{r=R_{3}+h_{3} / 2}-T_{c}\right) ; \\
& \left.\lambda_{k}\left(T_{k}\right) \frac{\partial T_{k}}{\partial s}\right|_{s= \pm L}=\mp \vartheta\left(\left.T_{k}\right|_{s=L}-T_{c}\right),
\end{aligned}
$$

где $\vartheta$ - коэффициент теплоотдачи в окружающую среду; $R_{i}$ и $h_{i}$ - соответственно радиусы срединных поверхностей и толщины металлических слоёв 1 и 3 (см. рис. 1); $L$ - половина длины оболочки; $T_{c}$ - температура окружающей среды.

Для срединного сечения граничное условие имеет вид

$$
\left.\frac{\partial T_{k}}{\partial s}\right|_{s=0}=0
$$

В качестве начального условия принимается равномерное распределение температуры, равной температуре заливки стекломассы:

$$
T_{k}(s ; r ; 0)=T_{m} .
$$


Для численного решения уравнений теплопроводности (1) при условиях сопряжения слоёв (2), граничных условиях (3), (4) и начальном условии (5) разработан алгоритм на языке программирования Delphi с использованием сеточного метода.

Численное решение позволяет моделировать различные условия теплоотдачи на внутренней и внешней обшивках и учитывать потери тепла на торце. Путём введения эффективного коэффициента теплоотдачи можно учесть влияние излучения на теплообмен и неоднородность теплообмена по толщине стеклослоя. Может быть определена температура поверхности контакта металлических обшивок со стекломассой в любой момент времени и время остывания стекломассы до характерных значений температуры: спаивания с металлическими обшивками, стеклования стекломассы и окружающей среды. Тем самым определяются основные параметры температурного режима формирования трёхслойной цилиндрической оболочки из стеклометаллокомпозита.

2. Пример расчёта цилиндрической оболочки из стеклометаллокомпозита. В качестве примера рассмотрим модельную цилиндрическую оболочку, состоящую из наружных стальных обшивок (сталь 40Х13) и внутреннего стеклянного слоя (техническое стекло типа ВВ). Сталь 40X13 сохраняет упругие свойства до температуры стеклования стекла $\left(550^{\circ} \mathrm{C}\right)$. Поэтому рассматриваемый пример позволяет оценить предельные значения остаточных напряжений на поверхностях сопряжения слоёв композитной оболочки.

Параметры обшивок из стали 40X13:

- коэффициенты теплопроводности $\lambda_{1}, \lambda_{3}, \mathrm{~B}$ т $/\left(\right.$ м $\left.\cdot{ }^{\circ} \mathrm{C}\right)$ :

$$
\lambda_{1}=\lambda_{3}=\left\{\begin{array}{cc}
24,9+0,011 T, & 20^{\circ} \mathrm{C} \leqslant T \leqslant 400^{\circ} \mathrm{C} \\
29,1, & 400^{\circ} \mathrm{C} \leqslant T \leqslant 600^{\circ} \mathrm{C} \\
34,51-0,009 T, & 600^{\circ} \mathrm{C} \leqslant T \leqslant 1200^{\circ} \mathrm{C}
\end{array}\right.
$$

- коэффициенты температуропроводности $a_{1}, a_{3}, \mathrm{~m}^{2} / \mathrm{c}$ :

$$
a_{1}=a_{3}=\left\{\begin{array}{cr}
\left(7+0,0016 T-0,85 \cdot 10^{-5} T^{2}\right) \cdot 10^{-6}, & 20^{\circ} \mathrm{C} \leqslant T \leqslant 700^{\circ} \mathrm{C} ; \\
(-0,757+0,0066 T) \cdot 10^{-6}, & 700^{\circ} \mathrm{C} \leqslant T \leqslant 1200^{\circ} \mathrm{C} .
\end{array}\right.
$$

Параметры технического стекла типа ВВ:

- коэффициент теплопроводности $\lambda_{2}, \mathrm{~B} /\left(\mathrm{m} \cdot{ }^{\circ} \mathrm{C}\right)$ :

$$
\lambda_{2}=0,87+0,00145 T
$$

- коэффициент температуропроводности $a_{2}, \mathrm{~m}^{2} / \mathrm{c}$ :

$$
a_{2}=\left(0,412+0,578 T-0,45 \cdot 10^{-5} T^{2}\right) \cdot 10^{-6} ;
$$

- коэффициент теплоотдачи $\vartheta$ принят равным $5,6 \mathrm{Bт} /\left(\mathrm{m}^{2} \cdot{ }^{\circ} \mathrm{C}\right)$;

- количество тепловой энергии $\delta q$, требующейся для обеспечения соединения стекла с металлическими обшивками, принято следующим: $\delta q^{-}=$ $=\delta q^{+}=0$ Дж. 
Геометрические размеры оболочки: радиус срединой поверхности стеклянного слоя $R_{2}=1 \mathrm{м}$, его толщина $h_{2}=0,1 \mathrm{м} ;$ радиус срединной поверхности внутреннего металлического слоя $R_{1}=0,9495$ м, его толщина $h_{1}=0,001$ м; радиус срединной поверхности внешнего металлического слоя $R_{3}=1,055 \mathrm{~m}$, его толщина $h_{3}=0,01$ м; половины длины оболочки $L=4$ м.

На рис. 2 представлен график остывания стеклянного слоя стеклометаллокомпозита. Из него следует, что время остывания оболочки до температуры размягчения стекла составляет примерно 6,5 часа. Температура стеклования достигается через 8,5 часа после начала остывания. При температуре стеклования в течение $\Delta t=3,5$ часа производится отжиг, который задерживает охлаждение оболочки и обеспечивает полную релаксацию напряжений и стабилизацию физико-химических свойств стекла. Полное время остывания достигается за 60 часов, сократить его можно за счёт принудительного охлаждения на заключительном этапе формирования оболочки.

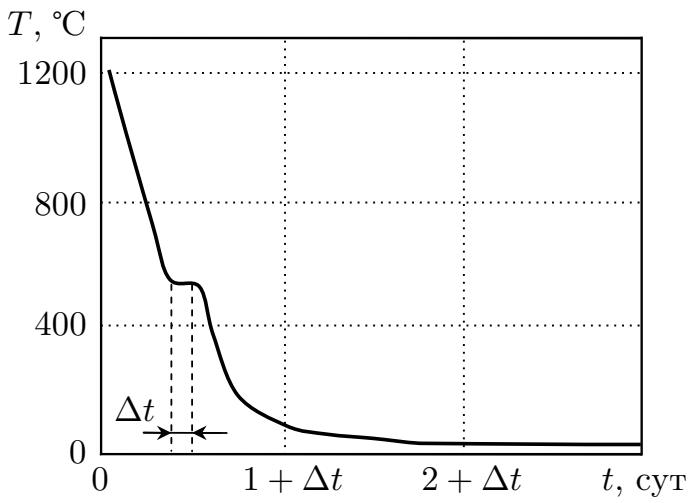

Рис. 2. Средние значения температур в стеклянном слое $(\Delta t=3,5$ ч)

3. Определение остаточных напряжений. Из-за различия коэффициентов температурного расширения металлических обшивок и стеклянного слоя в оболочке при остывании появляются остаточные напряжения, которые определяются после отжига оболочки при начальных условиях полного отсутствия напряжений, устранённых вследствие их релаксации при отжиге [3]. Для определения остаточных напряжений использован метод физической дискретизации трёхмерных задач механики оболочек [4] и специально разработанный аналитический метод решения поставленной задачи [3].

Деформирование каждого слоя оболочки рассматривается в местной системе координат при совмещении основных координатных поверхностей со срединными поверхностями слов. Основные координатные поверхности являются круговыми цилиндрическими. За их координаты приняты расстояние $s$ вдоль образующей, отсчитываемое от срединного поперечного сечения, и угол $\phi$ между начальным и текущим радиальными направлениями. Третьи координаты для каждого $z_{k}$ отсчитываются по нормали к основным координатным поверхностям в направлении внешней нормали $\left(z_{k}=r-R_{k}\right)$. Такие координаты позволяют свести краевую задачу механики оболочек к рассмотрению деформирования срединной поверхности и тем самым понизить трудоёмкость решения не менее чем на три порядка.

Математическая модель деформирования цилиндрической оболочки из 
стеклометаллокомпозита включает фундаментальные уравнения, пригодные для каждого слоя оболочки, уравнения состояния, характеризующие индивидуальные механические и теплофизические свойства материала слоев, условия сопряжения слоев и краевые условия. Фундаментальные уравнения состоят из уравнений:

- равновесия

$$
\begin{gathered}
\frac{d N_{11}}{d s}=-\left(\sigma_{31}^{+}-\sigma_{31}^{-}\right) ; \\
\frac{d^{2} M_{11}}{d s^{2}}-\frac{N_{22}}{R}=-\frac{h}{2} \frac{d}{d s}\left(\sigma_{31}^{+}+\sigma_{31}^{-}\right)-\left(\sigma_{33}^{+}-\sigma_{33}^{-}\right) ; \\
Q_{1}=\frac{d M_{11}}{d s}+\frac{h}{2}\left(\sigma_{31}^{+}+\sigma_{31}^{-}\right) ;
\end{gathered}
$$

- геометрии

$$
\varepsilon_{11}=\frac{d u}{d s}+z \frac{d \psi_{1}}{d s} ; \quad \varepsilon_{22}=\frac{w}{R} ;
$$

- условий сопряжения слоев

$$
\begin{aligned}
u^{(1)}+h_{1} / 2 \psi_{1}^{(1)}=u^{(2)}-h_{2} / 2 \psi_{1}^{(2)} ; & u^{(2)}+h_{2} / 2 \psi_{1}^{(2)}=u^{(3)}-h_{3} / 2 \psi_{1}^{(3)}, \\
w^{(1)}+\Delta w_{1}^{+}=w^{(2)}+\Delta w_{2}^{-} ; & w^{(2)}+\Delta w_{2}^{+}=w^{(3)}+\Delta w_{3}^{-}
\end{aligned}
$$

- граничных условий

$$
\begin{array}{cc}
\text { при } s=0, s=L: & Q_{1}^{(k)}=0 ; \quad N_{11}^{(k)}=0 ; \quad M_{11}^{(k)}=0 ; \\
& \sigma_{31}^{-(1)}=\sigma_{33}^{-(1)}=\sigma_{31}^{+(3)}=\sigma_{33}^{+(3)}=0,
\end{array}
$$

где $N_{11}$ и $N_{22}$ - удельные тангенциальные силы, отнесённые к единице длины в направлении образующей и окружном направлении соответственно; $M_{11}$ удельный изгибающий момент, отнесённый к единице длины в направлении образующей; $\sigma_{31}^{-}$и $\sigma_{33}^{-}$-соответственно касательное и нормальное напряжения, действующие на нижней лицевой поверхности расчётного слоя; $\sigma_{31}^{+}$и $\sigma_{33}^{+}$- соответственно касательное и нормальное напряжения, действующие на верхней лицевой поверхности расчётного слоя; $Q_{1}$ - удельная поперечная сила, отнесённая к единице длины; $u$ и $w$-перемещения срединной поверхности вдоль осей $s$ и $z$ соответственно, зависящие только от $s ; \psi_{1}-$ угол поворота нормали к срединной поверхности; $\varepsilon_{11}$ - нормальные деформации в направлении образующей цилиндрического слоя, $\varepsilon_{22}$ - нормальные деформации в окружном направлении; $R$ - радиус срединной поверхности; $h$-толщина слоя; $L$ - длина половины оболочки; $\Delta w$-дополнительные прогибы поверхностей сопряжения слоев, вызванные стесненной частью поперечных деформаций:

$$
\Delta w_{i}^{ \pm}=\frac{1}{E_{i}} \int_{0}^{ \pm h_{i} / 2} \sigma_{33}^{(i)} d z .
$$

Фундаментальные уравнения дополняются уравнениями состояния материала: 
- для внешних обшивок

$$
\begin{aligned}
& \sigma_{11}=\frac{E}{1-\nu^{2}}\left[\left(\varepsilon_{11}+\nu \varepsilon_{22}\right)-(1+\nu) \alpha^{+} \Delta T\right], \\
& \sigma_{22}=\frac{E}{1-\nu^{2}}\left[\left(\varepsilon_{22}+\nu \varepsilon_{11}\right)-(1+\nu) \alpha^{+} \Delta T\right] ;
\end{aligned}
$$

- для стеклянного слоя

$$
\begin{aligned}
\sigma_{11} & =\frac{E}{1-\nu^{2}} \exp (-\mu t) \int_{t_{0}}^{t}\left[\left(\dot{\varepsilon}_{11}+\nu \dot{\varepsilon}_{22}\right)-(1+\nu) \dot{\alpha}^{+} \Delta T\right] \exp (\mu t) d t, \\
\sigma_{22} & =\frac{E}{1-\nu^{2}} \exp (-\mu t) \int_{t_{0}}^{t}\left[\left(\dot{\varepsilon}_{22}+\nu \dot{\varepsilon}_{11}\right)-(1+\nu) \dot{\alpha}^{+} \Delta T\right] \exp (\mu t) d t ;
\end{aligned}
$$

где $\sigma_{11}$ - нормальные напряжения в направлении образующей цилиндрического слоя; $\sigma_{22}$ - нормальные напряжения в окружном направлении; $E-$ осреднённый по температуре модуль упругости; $\nu$ - коэффициент Пуассона; $\alpha^{+}$- осреднённый по температуре коэффициент температурного расширения; $\Delta T$ - перепад температур; $T_{0}, T$ - начальная и конечная температура материалов слоёв; точкой сверху обозначены производные по времени; $\mu=$ $=E /(2(1+\nu) \eta)$ - коэффициент, учитывающий вязко-упругие свойства стеклянного слоя; $\eta$ - коэффициент динамической вязкости стекла.

Процесс деформирования является симметричным относительно среднего поперечного сечения оболочки и осесимметричным относительно оси симметрии, поэтому рассматривается только половина длины оболочки. При этом перемещения, деформации и напряжения оказываются независимыми от координаты $\phi$.

Удельные силы и изгибающие моменты можно определить через перемещения:

$$
\begin{gathered}
Q_{1}=\int_{h}\left(1+\frac{z}{R}\right) \sigma_{13} d z=\frac{E h}{2(1+\nu)} \gamma_{1} \\
N_{11}=\int_{h}\left(1+\frac{z}{R}\right) \sigma_{11} d z=\frac{E h}{1-\nu^{2}}\left[\frac{d u}{d s}+\nu \frac{w}{R}+\frac{h^{2}}{12 R} \frac{d \psi_{1}}{d s}\right]-\sigma_{t} h \\
N_{22}=\int_{h} \sigma_{22} d z=\frac{E h}{1-\nu^{2}}\left[\frac{w}{R}+\nu \frac{d u}{d s}\right]-\sigma_{t} h \\
M_{11}=\int_{h}\left(1+\frac{z}{R}\right) z \sigma_{11} d z=\frac{E h^{3}}{12\left(1-\nu^{2}\right)}\left[\frac{d \psi_{1}}{d s}+\frac{1}{R} \frac{d u}{d s}\right]-\sigma_{t} \frac{h^{3}}{12 R} \\
M_{22}=\int_{h} z \sigma_{22} d z=\frac{E \nu h^{3}}{12\left(1-\nu^{2}\right)} \frac{d \psi_{1}}{d s},
\end{gathered}
$$

где

$$
\sigma_{t}=\frac{E}{1-\nu} \alpha^{+} \Delta T
$$

- температурная составляющая напряжений.

Для решения поставленной задачи разработан аналитический метод, сущность которого заключается в последовательной подстановке всех функций 
перемещений и силовых факторов в условия сопряжения слоёв (6). В результате решение сводится к отысканию касательных и радиальных напряжений на границе сопряжения нижней $\left(\tau_{13}^{-}\right.$и $\left.\tau_{33}^{-}\right)$и верхней $\left(\tau_{13}^{+}\right.$и $\left.\tau_{33}^{+}\right)$металлической обшивки со стеклянным слоем [3].

Результаты определения остаточных напряжений, возникших после остывания на поверхностях сопряжения слоёв для примера, рассмотренного в п. 2, представлены на рис. 3 и 4. Возле торцов оболочки наблюдается всплеск касательных напряжений. Максимальное значение касательного напряжения равно 4,6 МПа, отрывного - 20 МПа, сжимающего - 18 МПа [3]. Эти значения напряжений существенно меньше пределов прочности сопрягаемых материалов композитной оболочки.

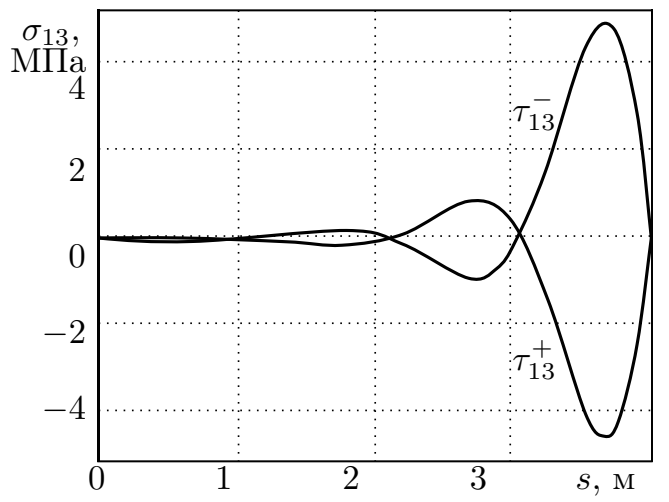

Рис. 3. Распределение касательных напряжений по длине цилиндрической оболочки

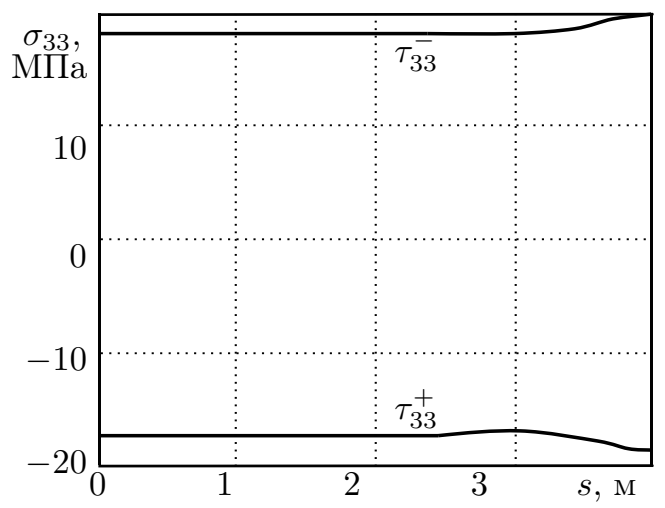

Рис. 4. Распределение радиальных напряжений по длине цилиндрической оболочки

Заключение. В результате проведённых исследований разработаны методы расчёта и программы, определяющие температурный режим процесса формирования цилиндрической оболочки из стеклометаллокомпозита и расчёт остаточных напряжений на поверхностях сопряжения слоёв и в оболочке в целом.

$\mathrm{K}$ настоящему времени установлены реальная возможность получения наноструктуры стекла в составе стеклометаллокомпозита и надёжность соединения металлических обшивок со стеклянным слоем [5]. На возможные способы изготовления различных изделий из стеклометаллокомпозита получены 
7 патентов РФ. Три последних патента на изготовление цилиндрической оболочки представлены в списке литературы [6-8].

Работа выполнена при поддержке РФФИ (проект № 09-08-98516-р восток а).

\title{
БИБЛИОГРАФИЧЕСКИЙ СПИСОК
}

1. Пикуль В.В. Перспективы создания слоистого композита на основе стекломатериалов // Перспективные материалы, 1999. - № 1. - С. 61-64.

2. Пикуль В. В. К созданию композиционного наноматериала на основе стекла // Перспективные материалы, 2008. - № 3. - С. 78-81.

3. Пикуль В. В., Ратников А. А. Математическое моделирование деформирования цилиндрической оболочки из стеклометаллокомпозита при её изготовлении // Перспективные материалы, 2007. - №3. - С. 10-15.

4. Пикуль В. В. Механика оболочек. - Владивосток: Дальнаука, 2009. - 536 с.

5. Пикуль B. В., Гончарук В. К. Композиционный наноматериал на основе стекла - стеклометаллокомпозит / В сб.: Все материаль. Энциклопедичный справочник. - № 6 . М.: Науки технологии, 2009. - C. 5-10.

6. Пикуль В. В., Наумов Л. А., Гончарук В. К. Патент на изобретение № 2337036. - Способ изготовления цилиндрической оболочки прочного корпуса подводного аппарата // Бюл. изобр., 2008. - № 30 .

7. Пикуль В. В. Патент на изобретение № 2361770. - Способ изготовления цилиндрической оболочки прочного корпуса подводного аппарата // Бюл. изобр., 2009. - № 20.

8. Пикуль В. В. Патент на изобретение № 2361771. - Способ изготовления цилиндрической оболочки прочного корпуса подводного аппарата // Бюл. изобр., 2009. - № 20.

Поступила в редакцию 01/VII/2010;

в окончательном варианте - 06/IX/2010.

MSC: 74K25, 74E30, 74S30

\section{MATHEMATICAL MODELLING OF GLASS-METALIC ENVELOPE THREE-LAYER CYLINDRICAL SHELL PROCESS FORMATION}

\author{
V. V. Pikul ${ }^{1}$, A.A. Ratnikov ${ }^{2}$ \\ 1 Institute of Marine Technology Problems, Far East Branch of RAS, \\ 5 a, Sukhanov st., Vladivostok, 690950, Russia. \\ 2 Far Eastern State Technical University, \\ Institute of Natural Science, Faculty of Mechanics and Mathematics, \\ 10, Pushkinskaya st., Vladivostok, 690600, Russia. \\ E-mails: pikulv@mail.ru, ratnikov2003@pisem.net
}

The results of cylindrical layered silicate glass based material shell manufacturing process mathematical modeling are presented in the article. Problems of cooling of a composit shell and axisymmetric deformation in the course of its formation are considered. The received results testify the possibility of manufacturing of three-layer cylindrical glass-metalic envelope shells which can be used for creation of strong cases for deepwater technical devices. Limiting values of residual pressure on interface surfaces of metal coverings with a glass layer which it is essential less strength of connected materials of a composit cover are received.

Key words: cylindrical shell, glass-metalic envelope, namomaterial, temperature problem, heat conductivity, numerical method, deformation problem, analytical method, residual stresses.

Original article submitted 01/VII/2010; revision submitted 06/IX/2010.

Vladimir V. Pikul (Dr. Sci. (Phys. \& Math.)), Head of Lab., Lab. of Deepwater Engineering Problems of Strength. Alexander A. Ratnikov, Senior Lecturer, Dept. of Applied Mathematics $\&$ Mechanics. 